\title{
The Research on Image Binarization Based on Automated Line-heating
}

\author{
Hao $L^{1, a}$, Jianhua Yang ${ }^{2, b}$ and Wei $L^{3, c}$ \\ ${ }^{1}$ School of Electronic and Information Engineering, Dalian University of Technology, Dalian 116024, \\ China; \\ ${ }^{2}$ School of Electronic and Information Engineering, Dalian University of Technology, Dalian 116024, \\ China; \\ ${ }^{3}$ School of Electronic and Information Engineering, Dalian University of Technology, Dalian 116024, \\ China. \\ alihaozdh@outlook.com, bjianhuay@dlut.edu.cn, 'luwei@dlut.edu.cn
}

Keywords: Automated line-heating, image binarization, thresholding method.

Abstract. In this paper, a thresholding algorithm combining local and global region is proposed in order to perfect the vision of line-heating robot, segment foreground objects and background accurately, eliminate environment effects. The thresholding method combines the advantages of both local and global threshold algorithm. First, we detect the edge region of foreground objects with Canny operator and get the gray-level histogram of edge region. Next, according to the gray-level histogram, we calculate a segment threshold with OTSU method. Finally, the gray image is converted into binary image according to the segment threshold. The experimental results show that the thresholding method combining local and global region is effective on segmenting foreground objects and background. Our method could insure the shape integrity of foreground objects as well.

\section{Introduction}

Modern shipbuilding technique is developing on a way of automatization. At present, line-heating technique [1] is used widely in processing double-curved hull plate. However, shipyards still depend on skilled workers to do this job. There are many disadvantages of manual process, such as poor working environment, long working hours and high rework rate [2]. To solve these problems, many shipyards are researching robots to process line-heating instead of manual operation.

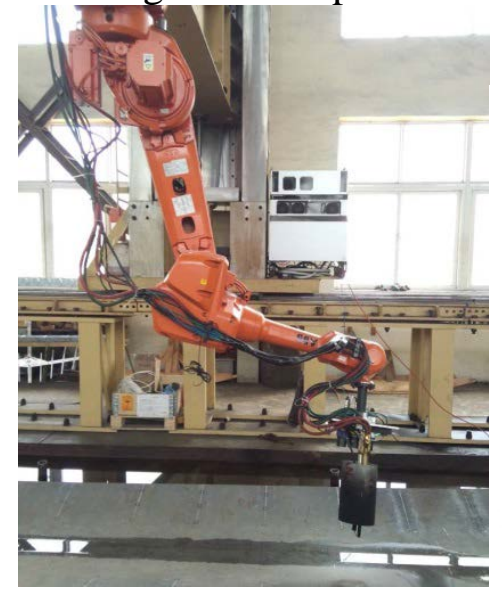

Fig. 1 Line-heating robot

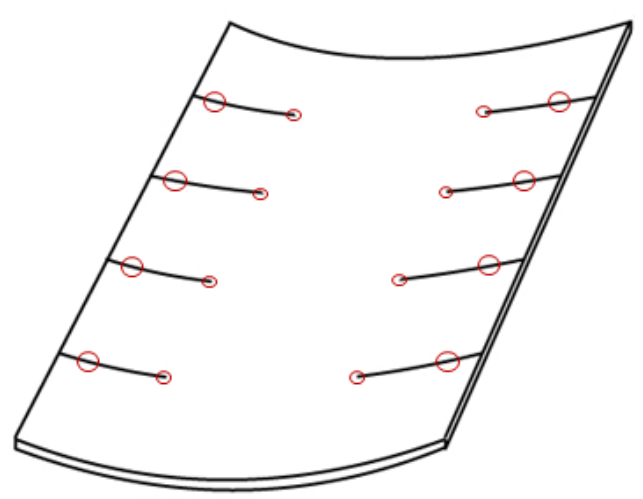

Fig. 2 Plate diagram

The background of this paper is developing visually guided robot used for line-heating process. Figure 1 shows the line-heating robot and Figure 2 shows a plate with marked flame paths. The robot is equipped with vision module. The vision module needs to segment foreground objects and background in an image with binarization transformation algorithms, so that the foreground objects could be recognized correctly. However, there are many outside factors influencing the transformation results, such as constantly changing light, floating dust, plate material. Due to these 
factors, the processed images may be too light or too dark and have lots of noise. To eliminate effects from outside factors and obtain clear binary image, an improved algorithm is proposed in this paper.

\section{Image binarization algorithms comparison}

Image binarization algorithm is used for converting gray image from 256 brightness levels into two brightness levels, which are 0 and 255. Converted images would show a clear black and white effect.

Processing gray images with thresholding is the most common algorithm in image binarization field. According to applied range, thresholding algorithms include global threshold algorithm and local threshold algorithm. Global threshold algorithm integrates the whole image information, just like histogram, to calculate the threshold. Local threshold algorithm calculates thresholds for each divided part and processes every part with its local threshold. Besides, according to the way to calculate threshold, there are some algorithms.

\section{Gray expectation algorithm}

Based on gray-level histogram, gray expectation algorithm [3] calculates binarization threshold according to an image's gray level expectation. The algorithm consists of the following steps:

(a) According to image's histogram, obtain maximum gray level $R_{\max }$ and minimum gray level $R_{\min }$. Then calculate the average value of $R_{\max }$ and $R_{\min }$

$$
T_{0}=\left(R_{\max }+R_{\min }\right) / 2
$$

(b) Calculate the gray expectation $T_{1}$ in the gray level range $\left[R_{\min }, T_{0}\right]$ and $T_{2}$ in the gray level range $\left[T_{0}+1, R_{\max }\right]$. Then get the average value of $T_{1}$ and $T_{2}$.

$$
T=\left(T_{1}+T_{2}\right) / 2
$$

(c) Let $p=\left|T-T_{0}\right|$. If $p$ is larger than the default difference, let $T_{0}=T$ and return to step (b). Conversely, $T$ is the binarization threshold calculated by gray expectation algorithm.

(d) Output $T$, the binarization threshold.

\section{OTSU method}

Proposed by Nobuyuki Otsu, OTSU method [4, 5] is also called Maximum Between Class Variance Method. The method's fundamental is selecting a gray level which could divide histogram values into two different groups with the largest variance. So the selected gray level is the image binarization threshold.

In a plate image, flame path marks' area proportion is quite small while background's is large. So it could generate serious difference with global threshold algorithm to calculate threshold. In the flame path marks' edge region, the proportions of both foreground objects and background are balanced. Consequently, a thresholding algorithm combining local and global region is proposed in this paper. This algorithm calculates threshold with local threshold algorithm and then converts the whole gray image into binary one with threshold calculated earlier.

Comparing with gray expectation algorithm, OTSU method is less readily influenced by noise. As a result, OTSU method is selected to calculate binarization threshold.

\section{A thresholding algorithm combining local and global region}

Concerning about features of original gray images, a thresholding algorithm combining local and global region is proposed in this article. The basic theory is to calculate binarization threshold according to the local area information from a gray image and apply the threshold to the whole image's binaryzation. And then the flame path marks could be segmented from background. The flowchart of this algorithm is shown in fugure 3. 


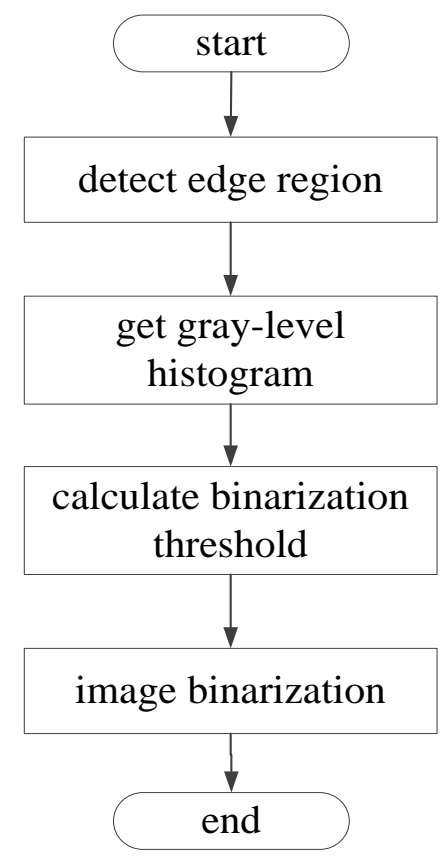

Fig. 3 Image binarization process

Canny operator [6] is used to detect image edge region in the new algorithm. Filtering operations are involved in the algorithm and could eliminate noise influence on edge detection. Besides, OTSU method quoted above is used to calculate binarization threshold. Briefly, the new algorithm calculates threshold according to the pixels in local region, and then apply the threshold to the binarization process of whole image. Consequently, the new algorithm is called a thresholding algorithm combining local and global region in this paper. The specific steps is shown below.

\section{(1) detect edge region with Canny operator}

Two-dimension Gaussian function is shown below.

$$
G(x, y)=\frac{1}{2 \pi \sigma^{2}} \exp \left(-\frac{x^{2}+y^{2}}{2 \sigma^{2}}\right)
$$

The gradient vector of Gaussian function above is

$$
\nabla G(x, y)=\left[\begin{array}{l}
\frac{\partial G}{\partial x} \\
\frac{\partial G}{\partial y}
\end{array}\right]
$$

The gradient vector $\nabla G(x, y)$ is the origin of Canny operator. Edge intensity is related to the modulus $|\nabla G(x, y) \times f(x, y)|$ and direction vector $\frac{\nabla G(x, y) \times f(x, y)}{|\nabla G(x, y) \times f(x, y)|}$. To simplify operations, two-dimension filter $\nabla G(x, y)$ is divided into two one-dimension filters

$$
\begin{aligned}
& \frac{\partial G}{\partial x}=-\frac{x}{2 \pi \sigma^{4}} \exp \left(-\frac{x^{2}}{2 \sigma^{2}}\right) \exp \left(-\frac{y^{2}}{2 \sigma^{2}}\right) \\
& \frac{\partial G}{\partial y}=-\frac{y}{2 \pi \sigma^{4}} \exp \left(-\frac{x^{2}}{2 \sigma^{2}}\right) \exp \left(-\frac{y^{2}}{2 \sigma^{2}}\right)
\end{aligned}
$$

Then apply convolution of two one-dimension filters and image matrix $f(x, y)$ on these input images

$$
E_{x}(x, y)=\frac{\partial G(x, y)}{\partial x} \cdot f(x, y)
$$




$$
E_{y}(x, y)=\frac{\partial G(x, y)}{\partial y} \cdot f(x, y)
$$

Given

$$
\begin{aligned}
& A(x, y)=\sqrt{E_{x}^{2}(x, y)+E_{y}^{2}(x, y)} \\
& \alpha(x, y)=\arctan \left(\frac{E_{y}(x, y)}{E_{x}(x, y)}\right)
\end{aligned}
$$

In the formulas above, $A(x, y)$ represents the edge intensity at the point $(x, y)$ in an image and $\alpha(x, y)$ represents the vertical edge direction.

Next, detect edge region with the double thresholds method and scan all the pixels in the image according to a high threshold value and a low threshold value. If a pixel's edge intensity is larger than the high threshold, the pixel is certainly in the edge region. If a pixel's edge intensity is in the range of high threshold and low threshold, and if there is an adjacent pixel whose edge intensity is larger high thrshold, then the pixel is in the edge region as well. In other situations, the pixel is not an edge point.

\section{(2) calculate binarization threshold}

Next, edge region's gray-level histogram is obtained according to edge pixels' gray value. In this paper, $R_{\max }$ represents the maximum gray value of an image while $R_{\min }$ represents the minimum gray value of an image. $T$ is the binarization threshold ( $R_{\min } \leq T \leq R_{\max }$ ) and could divides an image into two parts, foreground and background. The proportion of foreground objects is $w_{0}$ and foreground average gray is $u_{0}$. The proportion of background is $w_{1}$ and background average gray is $u_{1}$. Accordingly, the average gray of an image is

$$
u_{T}=w_{0} \times u_{0}+w_{1} \times u_{1}
$$

So $u_{T}$ is binarization threshold that segments foreground and background. The gray value variance of foreground and background is

$$
\sigma^{2}=w_{0} \times\left(u_{0}-u_{T}\right)^{2}+w_{1} \times\left(u_{1}-u_{T}\right)^{2}
$$

The gray value variance is the measurement of difference between foreground and background. The larger variance $\sigma^{2}$ is, the more significant difference between foreground and background is.

The variance function is

$$
f\left(u_{T}\right)=w_{0} \times\left(u_{0}-u_{T}\right)^{2}+w_{1} \times\left(u_{1}-u_{T}\right)^{2}
$$

When the variance function $f\left(u_{T}\right)$ gets the maximum value, the threshold $T$ corresponding to $u_{T}$ is the best binarization threshold.

\section{(3) Image binarization based on selected threshold}

Finally, make the pixels traversing in the whole image. A pixel's gray value would change to 255 if the gray value of a pixel $f(x, y)$ is larger than or equal to $T$. On the contrary, the value would be 0 if the gray value of a pixel $f(x, y)$ is smaller than $T$. The calculating process of pixels' value could be summed as a set of formulas below.

$$
f(x, y)=\left\{\begin{array}{cc}
0 & f(x, y) \leq T \\
255 & f(x, y) \geq T
\end{array}\right.
$$

Accordingly, the frame path markings and background could be segmented.

\section{Experimental results and analysis}

Image binarization algorithms could be distinguished based on segmentation effect. In order to compare the image binarization algorithms mentioned before, gray expectation algorithm, OTSU method and the new algorithm proposed in this paper are used to do binarization transformation and then the results are comparied. Matlab 2009Ra is the programming platform of the experiments in this paper. And the experimental subjects are gray images photoed at the line-heating workshop of Dalian 
Shipbuilding Industry Group. The gray images are shown in figure 4, which contains three original images and processed images. These three original images are photoed respectively on different condition of bright light, normal light and dim light, and the foreground objects is large circles.
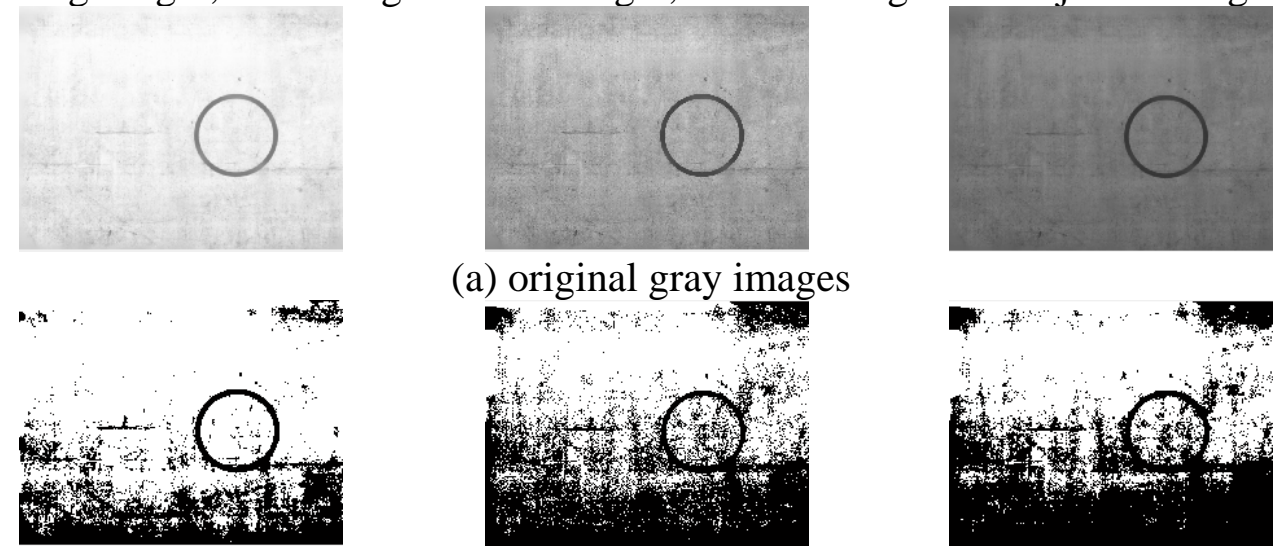

(a) original gray images
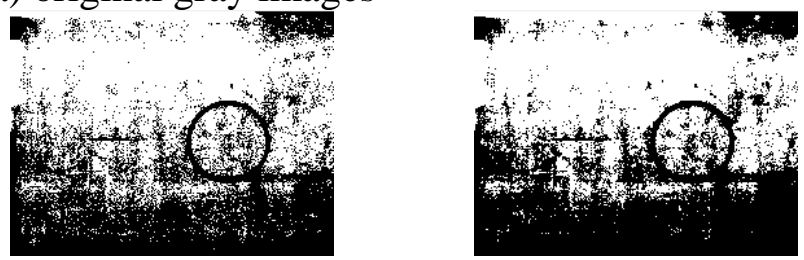

(b) processed images with gray expectation algorithm
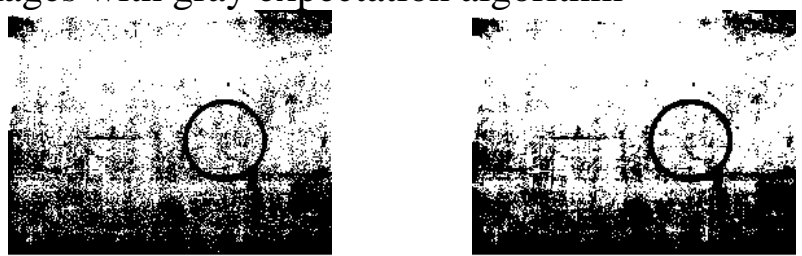

(c) processed images with OTSU method
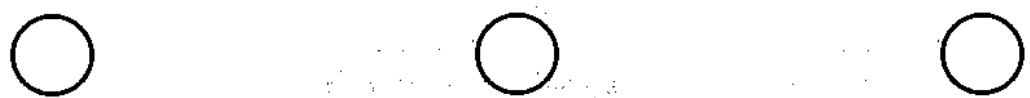

(d) processed images with algorithm proposed in this paper

Figure 4 images with large circle markings

From the experiment results, we can conclude:

(1) The binarization results of gray expectation algorithm contain lots of black background region, so this algorithm could not segment foreground objects and background effectively.

(2) OTSU method has clear processing effect in bright light. However, black background in the processed images would arise when light turns to dim.

(3) The algorithm proposed in this paper could not only highlight foreground objects from background whatever the light condition is, but also keep the shape of foreground objects.

In summary, the thresholding algorithm combining local and global region could segment foreground objects and background effectively. Consequently, this algorithm shows good application effect in image binarization of line-heating robot's vision.

The binary images obtained with gray expectation algorithm and OTSU method are unclear. The reasons are that the area proportion of flame path marks in an image is little and the level difference between foreground and background is small. Therefore, it is difficult to calculate a proper binarization threshold with these two algorithms. However, based on the features of the gray images to be processed, the algorithm proposed in this paper is based on the features of the gray images to be processed and calculates threshold according to the edge region of foreground objects. In the edge region, the proportions of both foreground objects and background are balanced. So the threshold could be easy to calculate. Otherwise, the algorithm proposed in this paper involves filtering operations, which could eliminate noise's influence.

\section{Summary}

In this paper, several image binarization algorithms, especially gray expectation algorithm and OTSU method, are analyzed and compared. However, these algorithms could hardly process the 
images of line-heating plates well. Combining the merits of OTSU method and local threshold algorithm, a thresholding algorithm combining local and global region is proposed in this paper. This algorithm could not only segment foreground objects and background effectively, but also insure the shape integrity of foreground objects. Clear binarization results could make it easy to realize other functions of robot's vision module.

\section{References}

[1] Wang Ji, Liu Yujun, The Visual Detecting and Control Research of Line-Heating Automated Processing Equipment. Dalian University of Technology, 2014

[2] Xiong Yuzhu, Yang Jianhua, The Study and Design of Arm Robot for Hull Shaped Plate Forming by Line Heating. Dalian University of Technology, 2006

[3] Zhang Qi, Dong Tian, Image Binarization Algorithm Combined with Edge Detection. Jilin University, 2011

[4] OTSU N. A threshold selection method from gray level histograms[J]. IEEE Trans on SMC, 1979, 9(1): 62-69.

[5] Bolan Su, Shijian Lu, Tan, C.L., Combination of Document Image Binarization Techniques. Document Analysis and Recognition (ICDAR), 2011 International Conference

[6] Luo Tao, Zheng Xi-feng, Ding Tie-fu, Improved Self-adaptive Threshold Canny Edge Detection. Opto-Electronic Engineering, 2009 